\title{
A systematic review of prognostic factors predicting survival in patients with spinal bone metastases
}

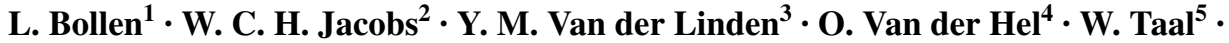 \\ P. D. S. Dijkstra ${ }^{1}$
}

Received: 23 April 2017 / Revised: 2 September 2017 / Accepted: 30 September 2017 / Published online: 24 October 2017

(C) The Author(s) 2017. This article is an open access publication

\begin{abstract}
Purpose For the selection of treatment in patients with spinal bone metastases (SBM), survival estimation plays a crucial role to avoid over- and under-treatment. To aid clinicians in this difficult task, several prediction models have been developed, consisting of many different risk factors. The aim of this systematic review was to identify prognostic factors that are associated with survival in patients with SBM to support development of predictive models.

Methods A systematic review was performed with focus on prognostic factors associated with survival in patients with SBM. Two reviewers independently selected studies for inclusion and assessed the risk of bias. A level of evidence synthesis was performed for each prognostic factor. Inter-observer agreement for the risk of bias assessment was determined by the kappa-statistic.
\end{abstract}

Electronic supplementary material The online version of this article (doi:10.1007/s00586-017-5320-3) contains supplementary material, which is available to authorized users.

L. Bollen

1.bollen@amc.nl

1 Department of Orthopedic Surgery, Leiden University Medical Center, Albinusdreef 2, P.O. Box 9600, 2300 RC Leiden, The Netherlands

2 Department of Neurosurgery, Leiden University Medical Center, Leiden, The Netherlands

3 Department of Radiotherapy, Leiden University Medical Center, Leiden, The Netherlands

4 Integraal Kankercentrum Nederland, Utrecht, The Netherlands

5 Department of Neuro-Oncology/Neurology, Erasmus MC Cancer Institute, Rotterdam, The Netherlands
Results After screening, 142 full-text articles were obtained, of which 22 met the eligibility criteria. A total of 43 different prognostic factors were investigated in the included studies, of which 17 were relevant to pre-treatment survival estimation. The prognostic factors most frequently associated with survival were the primary tumor and the performance status. The prognostic factors most frequently not associated with survival were age, gender, number and location of the SBM and the presence of a pathologic fracture. Conclusions Prognostication for patients with SBM should be based on an accurate primary tumor classification, combined with a performance score. The benefit of adding other prognostic factors is doubtful.

Keywords Spinal bone metastases - Prognostication · Systematic review

\section{Introduction}

Spinal bone metastases (SBM) are a frequently observed complication of malignant disease. Due to an increase in the survival times of patients with malignancies, the incidence of SBM is expected to rise over the years [1, 2]. The majority of symptomatic SBM are caused by breast, prostate and lung cancer (56-74\%) [3-5] and most commonly arise from the thoracic part of the spine (51-67\%) $[4,6]$. Patients present with pain due to destruction of bone tissue and/or neurologic complaints due to nerve root and/or spinal cord compression [7, 8]. Both radiotherapeutical and surgical interventions are effective in treating these symptoms. However, due to the relatively short life expectancy of these patients, overtreatment is a common problem. Therefore, an accurate estimation of survival plays a pivotal role in selecting the appropriate treatment. 
Survival estimation by clinicians has been shown to be too optimistic [9] and to provide an aid for this difficult task, several prediction models have been developed $[4,8$, $10,11]$. These models employ different sets of prognostic factors such as performance score, primary tumor and the presence of visceral metastases to stratify patients with SBM according to survival risk, enabling clinicians to select a more appropriate treatment. Several studies have been undertaken to assess the prognostic value of these models and the factors being used [12, 13]. The aim of this systematic review was to identify prognostic factors that are associated with survival in patients with spinal bone metastases in order to help guide development of predictive models.

\section{Methods}

This study was conducted according to the guidelines provided by the PRISMA statement.

\section{Search strategy}

The review protocol for this study was prospectively registered online at PROSPERO under registration number CRD42014006706 (http://www.crd.york.ac.uk/prospero). The search strategy was formulated in collaboration with a medical librarian to focus on the key terms survival, prognostic factors and spinal bone metastases, or variations thereof. The electronic databases Medline, Embase, Web of Science, Cochrane and Cinahl were searched from January 1999 up to September 2014. The complete search strategy is available as an online supplement.

\section{Eligibility criteria}

Both prospective and retrospective studies were eligible for inclusion if they met the following criteria: (1) Sample size of at least 100 patients with spinal bone metastases from solid tumors (i.e., no hematological malignancies); (2) The study did not focus on one single primary malignancy; (3) Prognostic factors for survival were assessed by means of a multivariate analysis; (4) Studies were published in the English, German or Dutch language. If studies were derived from identical databases, the most comprehensive study was selected; separately published subgroup analyses were disregarded. Eligibility of studies was assessed by two independent review authors (W. J. and L. B.). A consensus meeting was planned to resolve disagreements. If disagreements persisted, a third review author (P. D. S. D.) was consulted.

\section{Risk of bias}

The risk of bias was assessed according to the guidelines provided by Hayden et al. [14]. In short, six main sources of potential bias (study participation, study attrition, prognostic factor measurement, outcome measurement, confounding and analysis) were assessed using a 29 -item checklist. The six sources of bias were scored as being 'high; 3 points', 'moderate; 2 points', or 'low; 1 point'. Therefore, the total number of points for each study ranged from 6 to 18 , with a cut-off set at a maximum of $50 \%$ ( $\leq 9$ points) for distinguishing a low risk of bias study from a high risk of bias study. Two review authors (W. J. and L. B.) independently scored the risk of bias for each study. A consensus meeting was planned to resolve disagreements. If disagreements persisted, a third review author (P. D. S. D.) decided on the risk of bias.

\section{Data extraction}

The data extracted from eligible studies consisted of the design, source of funding, setting, sample size, duration, inclusion and exclusion criteria, all prognostic factors investigated, duration of follow-up and results. If similar prognostic factors were investigated by different means, they were combined for the level of evidence analysis. For instance, the prognostic factor Performance status was assessed by means of the Karnofsky score [15] or the Eastern Cooperative Oncology Group score [16]. Other combined prognostic factors were Neurologic deficit; consisting of the Frankel score [17] and ambulatory status, as well as the variable Primary tumor; consisting of several different sub classifications. Prognostic factors such as Age and Number or location of SBM were also combined irrespective of any differences in cut-off points used. Two review authors (O. H and L. B.) extracted the data on standardized forms. When consensus could not be reached, a third review author (P. D. S. D.) was consulted.

\section{Statistical analysis}

Statistical pooling of the results was not possible; therefore a level of evidence synthesis was performed for each prognostic factor. The levels of evidence were defined as follows: $[18,19]$.

- Strong evidence: consistent findings ( $\geq 75 \%)$ in multiple high-quality cohorts.

- Moderate evidence: consistent findings $(\geq 75 \%)$ in multiple cohorts, of which only one cohort was of high quality.

- Limited evidence: findings of one high-quality cohort, or consistent $(\geq 75 \%)$ findings in one or more low-quality cohorts. 


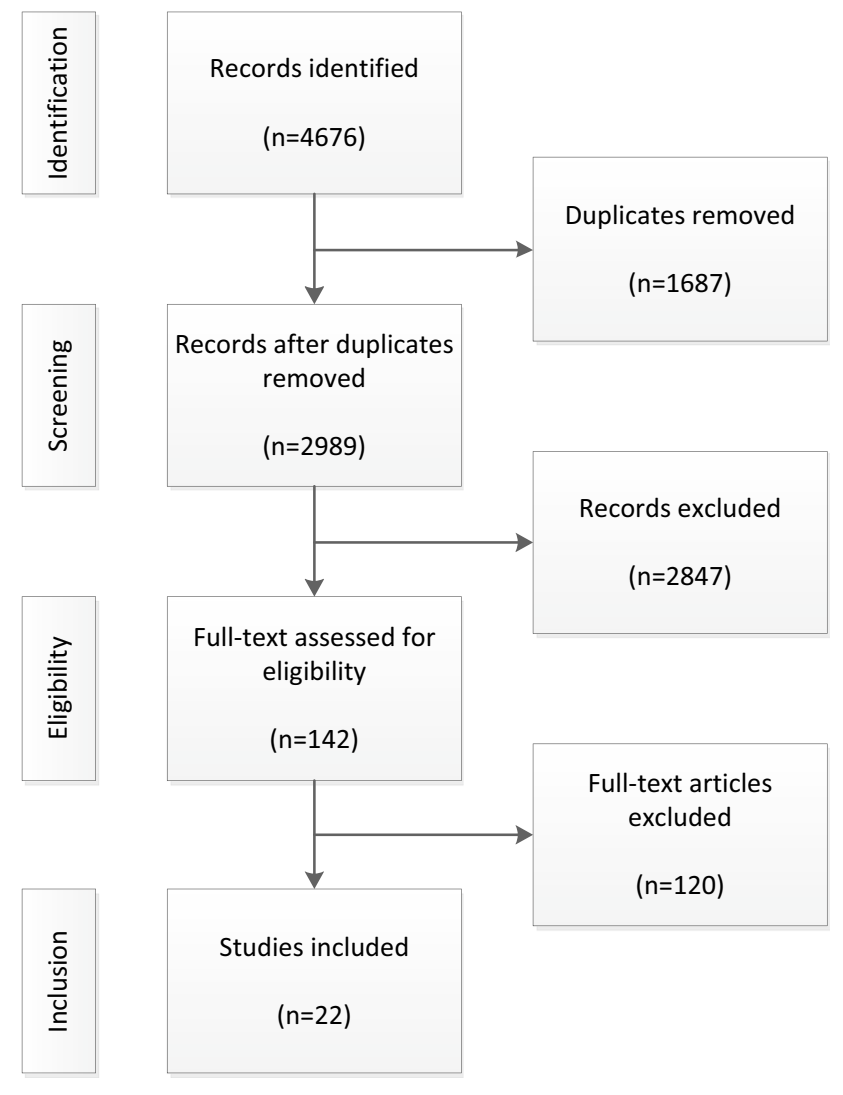

Fig. 1 Flowchart study selection process

- Inconclusive: inconsistent findings $(<75 \%)$ irrespective of study quality.

Inter-observer agreement for the risk of bias assessment was determined by the kappa-statistic [20]. All analyses were performed using SPSS 20.0, Armonk NY, IBM Corp.

\section{Results}

\section{Study selection}

The initial search yielded 4676 results (Medline $n=1996$; Embase $n=1389$; Web of Science $n=1092$; Cochrance $n=145$; Cinahl $n=54$ ). A total of 1687 duplicates were removed, leaving 2989 studies. After screening, 142 full-text articles were obtained, of which 120 did not meet the eligibility criteria: 33 studies were based on duplicate cohorts, 49 studies focused on a single primary tumor, 23 studies had less than 100 participants, 13 studies did not perform a multivariate analysis and two studies were excluded based on language. In total, 22 studies were included (Fig. 1) [3-6, 8, 21-37]. During the selection process, the reviewers disagreed on seven inclusions. Consensus was reached for all studies.

\section{Study characteristics}

The characteristics of the 22 included studies are presented in Table 1. Seven studies were based on prospectively collected data and the remaining 15 inclusions were retrospective studies. The population of seven studies consisted of surgically treated patients only, whereas five studies were based on patients treated with radiotherapy only. Nine studies consisted of a mixed population and the treatment details of one study were not reported. Duration of follow-up was reported in nine studies and ranged from 8 to 72 months. Median duration of the period of recruitment was 8 years and ranged from 1 to 20 years.

\section{Risk of bias}

Of the 22 included studies, agreement on the overall risk of bias was obtained for 18 (82\%). Consensus was reached for the remaining four studies. Inter-observer agreement for the overall risk of bias was substantial (kappa 0.62). Lower levels of agreement were mainly observed in the categories study participation (kappa 0.19) and confounding (kappa $-0.13)$.

\section{Prognostic factors levels of evidence}

A total of 43 different prognostic factors were investigated in the 22 included studies. Seven prognostic factors pertained to post-treatment details and four prognostic factors were specific to the received treatment of the patients in the cohort. These prognostic factors were therefore not considered relevant to pre-treatment estimation of survival and were excluded. Fifteen prognostic factors were analyzed only once. Because the level of evidence for these factors by definition could not exceed the category 'limited' they are not mentioned in the results, but are presented in an online supplement. The remaining seventeen prognostic factors are detailed in Table 2. Prognostic factors influencing survival in a certain study are mentioned in the column "positive association' and studied prognostic factors not influencing survival are mentioned in the column 'no association'.

Strong evidence for a positive association with survival was found for the primary tumor, performance status and the American Society of Anesthesiologists (ASA) classification [38]. Strong evidence for no association with survival was found for gender, age, number of spinal bone metastases, location of spinal bone metastases, and the presence of a pathologic fracture. The evidence was inconclusive for visceral metastases, neurologic deficit, the interval between diagnosis of the primary tumor and start of treatment for 
Table 1 Characteristics of the 22 included studies

\begin{tabular}{|c|c|c|c|c|c|c|c|c|}
\hline Study ID & Author, year, country & Study type & No. patients & $\%$ SUR/RTC & $\begin{array}{l}\text { Follow- } \\
\text { up } \\
\text { (months) }\end{array}$ & Period recruitment & $\begin{array}{l}\text { Duration } \\
\text { recruitment } \\
\text { (years) }\end{array}$ & Risk of Bias \\
\hline 1 & Helweg, 2000, Denmark & Prospective & 153 & $0 / 100$ & NR & 1996-1999 & 4 & High \\
\hline 2 & Riegel, 2002, Germany & Retrospective & 139 & NR & NR & 1990-1997 & 8 & High \\
\hline 3 & Linden, 2004, Netherlands & Prospective & 342 & $0 / 100$ & 16 & 1996-1998 & 3 & Low \\
\hline 4 & Hosono, 2005, Japan & Retrospective & 176 & $100 / 0$ & 23 & 1985-2001 & 16 & High \\
\hline 5 & $\begin{array}{l}\text { Ibrahim, 2008, Interna- } \\
\text { tional }\end{array}$ & Prospective & 223 & $100 / 0$ & NR & 2002-2003 & 2 & High \\
\hline 6 & Chi, 2009, USA & Prospective & 101 & $50 / 50$ & 8 & 1992-2002 & 11 & High \\
\hline 7 & Arrigo, 2011, USA & Retrospective & 200 & $100 / 0$ & NR & 1999-2009 & 11 & Low \\
\hline 8 & $\begin{array}{l}\text { Bartels, 2011, Interna- } \\
\text { tional }\end{array}$ & Retrospective & 567 & $9 / 91$ & NR & 1996-2008 & 13 & Low \\
\hline 9 & Mizumoto, 2011, Japan & Retrospective & 603 & $0 / 100$ & 19 & 2002-2007 & 5 & Low \\
\hline 10 & Park, 2011, Korea & Retrospective & 103 & $100 / 0$ & 26 & 2001-2008 & 8 & High \\
\hline 11 & Pointillart, 2011, France & Prospective & 142 & $83 / 17$ & NR & 2005-2007 & 3 & Low \\
\hline 12 & Wibmer, 2011, Austria & Retrospective & 254 & $25 / 75$ & 36 & 1998-2006 & 9 & Low \\
\hline 13 & Chao, 2012, USA & Retrospective & 174 & $0 / 100$ & 9 & 2006-2009 & 4 & High \\
\hline 14 & Kataoka, 2012, Japan & Retrospective & 143 & $50 / 50$ & 21 & 1990-2008 & 19 & Low \\
\hline 15 & Tancioni, 2012, Italy & Retrospective & 151 & $100 / 0$ & NR & 2004-2007 & 4 & High \\
\hline 16 & Yang, 2012, Korea & Retrospective & 217 & $100 / 0$ & NR & 2001-2009 & 9 & Low \\
\hline 17 & Balain, 2013, UK & Prospective & 199 & $52 / 48$ & NR & 2010-NR & NR & High \\
\hline 18 & Rades, 2013, Germany & Retrospective & 2029 & $0 / 100$ & NR & $1992-2011$ & 20 & High \\
\hline 19 & Tabouret, 2013, France & Retrospective & 148 & $100 / 0$ & 24 & 2004-2010 & 7 & Low \\
\hline 20 & Bollen, 2014, Netherlands & Retrospective & 1043 & $5 / 95$ & 72 & 2001-2010 & 10 & Low \\
\hline 21 & Morgen, 2014, Denmark & Prospective & 544 & $16 / 84$ & NR & 2011 & 1 & High \\
\hline 22 & Yeung, 2014, Hong Kong & Retrospective & 128 & $46 / 54$ & NR & 2001-2011 & 11 & High \\
\hline
\end{tabular}

SUR patients treated with surgery; $R T C$ patients treated with radiotherapy or conservative therapy; NR not reported

Table 2 Level of evidence for investigated prognostic factors. Numbers refer to study identification from Table 1

\begin{tabular}{|c|c|c|c|}
\hline Prognostic factor & Positive association & No association & Level of evidence \\
\hline Primary tumor & $\begin{array}{l}2, \mathbf{3}, 4,5,6, \mathbf{7}, \mathbf{8}, \mathbf{9}, 10, \mathbf{1 1}, \mathbf{1 2}, \mathbf{1 4}, 15,17 \\
\quad 18, \mathbf{2 0}, 21,22\end{array}$ & $13, \mathbf{1 6}, 19$ & Strong- $-86 \%$ \\
\hline Performance status & $2, \mathbf{3}, \mathbf{8}, \mathbf{9}, \mathbf{1 1}, \mathbf{1 2}, 13,17,18, \mathbf{1 9}, \mathbf{2 0}, 21,22$ & 14 & Strong-93\% \\
\hline ASA classification & 11,19 & & Strong-100\% \\
\hline Age & $6,9,13$ & $2, \mathbf{7}, 10, \mathbf{1 1}, \mathbf{1 2}, \mathbf{1 4}, 15, \mathbf{1 6}, 17,18, \mathbf{1 9}, 22$ & Strong- $80 \%$ \\
\hline Gender & $2, \mathbf{8}, 18$ & $\mathbf{7}, \mathbf{9}, 10, \mathbf{1 1}, \mathbf{1 2}, 13, \mathbf{1 4}, \mathbf{1 6}, 17, \mathbf{1 9}, 22$ & Strong- $-79 \%$ \\
\hline Number SBM & 21 & $2, \mathbf{1 1}, \mathbf{1 2}, \mathbf{1 4}, \mathbf{1 6}, 17,18, \mathbf{1 9}, \mathbf{2 0}, 22$ & Strong-91\% \\
\hline Location SBM & 8 & $2, \mathbf{7}, 10,11, \mathbf{1 6}, \mathbf{1 9}, \mathbf{2 0}, 22$ & Strong- $-89 \%$ \\
\hline Pathologic fracture & & $7,11,12$ & Strong-100\% \\
\hline Visceral metastases & $\mathbf{3}, \mathbf{9}, \mathbf{1 2}, \mathbf{1 4}, 15,17,18, \mathbf{1 9}, \mathbf{2 0}, 21,22$ & $2, \mathbf{7}, \mathbf{1 1}, 13$ & Inconclusive $-73 \%$ \\
\hline Neurologic deficit & $1,4,5,7,18,21$ & $2, \mathbf{1 2}, \mathbf{1 4}, \mathbf{1 6}, 17, \mathbf{1 9}, \mathbf{2 0}, 22$ & Inconclusive $-57 \%$ \\
\hline Interval diagnosis & $1,13, \mathbf{1 4}, 18$ & $15, \mathbf{1 6}$ & Inconclusive- $-67 \%$ \\
\hline Extraspinal bone metastases & $\mathbf{1 4}, 17,18$ & $\mathbf{1 2}, \mathbf{1 9}, \mathbf{2 0}, 21,22$ & Inconclusive $-63 \%$ \\
\hline Number bone metastases & 9,15 & 3, 21 & Inconclusive $-50 \%$ \\
\hline Pain & 4,11 & 14 & Inconclusive- $67 \%$ \\
\hline Sphincter function & 5 & 7,11 & Inconclusive- $67 \%$ \\
\hline Weight loss & 11 & 19 & Inconclusive $-50 \%$ \\
\hline Cardiovascular disease & 11 & 19 & Inconclusive- $-50 \%$ \\
\hline
\end{tabular}

Studies with a low risk of bias are in bold

ASA American Society of Anesthesiologists; SBM spinal bone metastases 
SBM, the presence of extraspinal bone metastases, total number of bone metastases, pain, sphincter function, weight loss and cardiovascular disease.

\section{Discussion}

In this systematic review, it is shown that the primary tumor, performance status and the ASA classification are associated with survival in patients with spinal bone metastases. Age, gender, number of SBM, location of SBM and the presence of a pathologic fracture are most likely not associated with survival.

Even though the eligibility criteria for this study were strict, several limitations were observed. Firstly, $68 \%$ of the included studies were conducted retrospectively and $55 \%$ of the studies consisted of populations that were treated either only with surgery or only with radiotherapy. This increased the risk of bias and therefore the quality of our results. Also, the way the studies evaluated the prognostic factors was heterogeneous and several different cut-off points were used.

The primary tumor was investigated as a potential prognostic factor in all but one study. Even though several different classifications were used, it was found to be associated with survival in $86 \%$ of the included studies, leaving no doubt that an accurate primary tumor classification is required for prognostication in patients with spinal bone metastases. The same applies to the performance status, with a positive association rate of $93 \%$. Irrespective of which specific score is used, it provides essential information for accurate prognostication. The ASA classification was investigated in two studies with a low risk of bias and both found a positive association with survival. Because it is rather similar to the performance status, it remains to be seen whether there is a significant benefit to including both factors in a prognostic model.

Age was found not to be associated with survival in $80 \%$ of the included studies. Considering the fact that a performance score generally provides a much better measure of a patient's health — and therefore prognosis - than age does, it is not unexpected that this variable is not significantly associated with survival in a multivariate analysis. Gender also was not associated with survival, with a rate of $79 \%$. Breast and prostate cancer make up a large percentage of the populations in the included studies. Since they are gender specific, it is likely that any potential effect of gender is corrected on multivariate analysis. Gender might play a role in SBM prognostication for certain primary cancers with a more even distribution, such as lung cancer. Number and location of the SBM, as well as the presence of pathologic fractures, had no effect on survival in the majority of the included studies. Even though these factors are important to consider when deciding on treatment-for instance determining extent of radiation field or levels of surgical fixation - they most likely do not need to be considered with respect to survival.

The rate of positive association for the presence of visceral metastases was $73 \%$, meaning the level of evidence was only just inconclusive. A recent study that stratified the risk factor analysis based on the primary tumor classification found that the effect of visceral metastases on survival changed between different tumor categories [4]. The survival of patients with a fast growing, aggressive tumor was not affected by visceral metastases, whereas patients with a slow growing tumor did have a significantly shorter survival when visceral metastases were present. The included low risk of bias studies that found a positive association had, on average, $10 \%$ more breast cancer patients in their population than studies that did not find an association. This difference in composition of the population might explain the different findings in these studies. The prognostic factor neurologic deficit consisted of two variables; ambulatory status and the Frankel classification. Because of the interaction with the performance status of a patient, this variable can be difficult to obtain, especially retrospectively. Even though it is an important variable to consider when deciding on type of treatment, it is unclear whether this variable plays a role in estimating survival.

A recently published meta-analysis by Luksanapruksa et al. [39] aimed to assess prognostic factors in patients with spinal bone metastases. Seventeen poor prognostic factors were identified, including the primary tumor classification and performance score. Contrary to the current study, however, Luksanapruksa et al. also found items such as number of SBM, presence of other bone metastases and ambulatory status important prognostic factors. This is most likely due to the fact that all studies by Rades et al. were included in the analysis. Since the same cohort was used repeatedly, the effects were somewhat amplified. Also, the large measure of heterogeneity found in most of the pooled risk factors indicate that a meta-analysis might not be the most ideal approach to addressing this topic.

In conclusion, prognostication for patients with SBM should be based on an accurate primary tumor classification, combined with a performance score. The added benefit of including the ASA classification should be studied further, as should the influence of visceral metastases and the presence of neurologic deficit.

\section{Compliance with ethical standards}

Conflict of interest The authors declare that they have no conflict of interest.

Open Access This article is distributed under the terms of the Creative Commons Attribution 4.0 International License (http://creativecommons.org/licenses/by/4.0/), which permits unrestricted use, 
distribution, and reproduction in any medium, provided you give appropriate credit to the original author(s) and the source, provide a link to the Creative Commons license, and indicate if changes were made.

\section{References}

1. Jemal A, Siegel R, Ward E (2007) Cancer statistics. CA Cancer J Clin 57:43-66

2. Loblaw DA, Perry J, Chambers A et al (2005) Systematic review of the diagnosis and management of malignant extradural spinal cord compression. J Clin Oncol 23(9):2028-2037

3. Mizumoto M, Harada H, Asakura $\mathrm{H}$ et al (2011) Radiotherapy for patients with metastases to the spinal column: a review of 603 patients at Shizuoka Cancer Center Hospital. Int J Radiat Oncol Biol Phys 79(1):208-213

4. Bollen L, van der Linden YM, Pondaag W et al (2014) Prognostic factors associated with survival in patients with symptomatic spinal bone metastases: a retrospective cohort study of 1043 patients. Neuro-oncology 16(7):991-998

5. Rades D, Hueppe M, Schild SE (2013) A score to identify patients with metastatic spinal cord compression who may be candidates for best supportive care. Cancer 119(4):897-903

6. Helweg-Larsen S, Sørensen PS, Kreiner S (2000) Prognostic factors in metastatic spinal cord compression: a prospective study using multivariate analysis of variables influencing survival and gait function in 153 patients. Int J Radiat Oncol Biol Phys 46(5):1163-1169

7. Bach F, Larsen BH, Rohde K et al (1990) Metastatic spinal cord compression. Occurrence, symptoms, clinical presentations and prognosis in 398 patients with spinal cord compression. Acta Neurochir 107(1-2):37-43

8. Van der Linden YM, Dijkstra PDS, Vonk E et al (2005) Prediction of survival in patients with metastases in the spinal column: results based on a randomized trial of radiotherapy. Cancer 103(2):320-328

9. Chow E, Harth T, Hruby G et al (2001) How accurate are physicians' clinical predictions of survival and the available prognostic tools in estimating survival times in terminally ill cancer patients? A systematic review. Clin Oncol 13(3):209-218

10. Tokuhashi $\mathrm{Y}$, Matsuzaki $\mathrm{H}$, Oda $\mathrm{H}$ et al (2005) A revised scoring system for preoperative evaluation of metastatic spine tumor prognosis. Spine 30(19):2186-2191

11. Tomita K, Kawahara N, Kobayashi T et al (2001) Surgical strategy for spinal metastases. Spine 26(3):298-306

12. Leithner A, Radl R, Gruber G et al (2008) Predictive value of seven preoperative prognostic scoring systems for spinal metastases. Eur Spine J 17(11):1488-1495

13. Bollen L, Wibmer C, Van der Linden YM et al (2016) Predictive value of six prognostic scoring systems for spinal bone metastases: an analysis based on 1379 patients. Spine 41(3):155-162

14. Hayden JA, Van der Windt DA, Cartwright JL et al (2013) Assessing bias in studies of prognostic factors. Ann Intern Med 158:280-286

15. Karnofsky DA, Abelmann WH, Craver LF et al (1948) The use of the nitrogen mustards in the palliative treatment of carcinoma. With particular reference to bronchogenic carcinoma. Cancer 1:634-656

16. Oken MM, Creech RH, Tormey DC et al (1982) Toxicity and response criteria of the Eastern Cooperative Oncology Group. Am J Clin Oncol 5(6):649-655

17. Frankel HL, Hancock DO, Hyslop G et al (1969) The value of postural reduction in the initial management of closed injuries of the spine with paraplegia and tetraplegia. I. Spinal Cord 7(3):179-192
18. Ariëns GA, Van Mechelen W, Bongers PM et al (2000) Physical risk factors for neck pain. Scand J Work Environ Health 26(1):7-19

19. Scholten-Peeters GG, Verhagen AP, Bekkering GE et al (2003) Prognostic factors of whiplash associated disorders: a systematic review of prospective cohort studies. Pain 104(1-2):303-322

20. Landis JR, Koch GG (1977) The measurement of observer agreement for categorical data. Biometrics 33(1):159-174

21. Riegel T, Schilling T, Sitter H et al (2002) Analysis of factors affecting the prognosis of vertebral metastases. Zentralbl Neurochir 63(1):2-6

22. Hosono N, Ueda T, Tamura D et al (2005) Prognostic relevance of clinical symptoms in patients with spinal metastases. Clin Orthop Relat Res 436:196-201

23. Ibrahim A, Crockard A, Antonietti P et al (2008) Does spinal surgery improve the quality of life for those with extradural (spinal) osseous metastases? An international multicenter prospective observational study of 223 patients. J Neurosurg J Neurosurg Spine 8(3):271-278

24. Chi JH, Gokaslan Z, McCormick P et al (2009) Selecting treatment for patients with malignant epidural spinal cord compression-does age matter?: results from a randomized clinical trial. Spine 34(5):431-435

25. Arrigo RT, Kalanithi P, Cheng I et al (2011) Predictors of survival after surgical treatment of spinal metastasis. Neurosurgery 68(3):674-681

26. Bartels RH, Feuth T, Rades D et al (2011) External validation of a model to predict the survival of patients presenting with a spinal epidural metastasis. Cancer Metastasis Rev 30(2):153-159

27. Park JH, Rhim SC, Jeon SR (2011) Efficacy of decompression and fixation for metastatic spinal cord compression: analysis of factors prognostic for survival and postoperative ambulation. J Korean Neurosurg Soc 50(5):434-440

28. Pointillart V, Vital J-M, Salmi R et al (2011) Survival prognostic factors and clinical outcomes in patients with spinal metastases. J Cancer Res Clin Oncol 137(5):849-856

29. Wibmer C, Leithner A, Hofmann G et al (2011) Survival analysis of 254 patients after manifestation of spinal metastases: evaluation of seven preoperative scoring systems. Spine 36(23):1977-1986

30. Chao ST, Koyfman SA, Woody N et al (2012) Recursive partitioning analysis index is predictive for overall survival in patients undergoing spine stereotactic body radiation therapy for spinal metastases. Int J Radiat Oncol Biol Phys 82(5):1738-1743

31. Kataoka M, Kunisada T, Tanaka M et al (2012) Statistical analysis of prognostic factors for survival in patients with spinal metastasis. Acta Med Okayama 66(3):213-219

32. Tancioni F, Navarria P, Pessina F et al (2012) Assessment of prognostic factors in patients with metastatic epidural spinal cord compression (MESCC) from solid tumor after surgery plus radiotherapy: a single institution experience. Eur Spine J 21:146-148

33. Yang SB, Cho W, Chang U (2012) Analysis of prognostic factors relating to postoperative survival in spinal metastases. J Korean Neurosurg Soc 51(3):127-134

34. Balain B, Jaiswal A, Trivedi JM et al (2013) The Oswestry Risk Index: an aid in the treatment of metastatic disease of the spine. Bone Jt J 95-B(2):210-621

35. Tabouret E, Cauvin C, Fuentes S et al (2015) Reassessment of scoring systems and prognostic factors for metastatic spinal cord compression. Spine J. 15(5):944-950

36. Morgen SS, Nielsen DH, Larsen CF et al (2014) Moderate precision of prognostic scoring systems in a consecutive, prospective cohort of 544 patients with metastatic spinal cord compression. J Cancer Res Clin Oncol 140(12):2059-2064

37. Yeung YN, Cheung KK, Lam TC (2014) A study of the predictive value of the modified Tokuhashi score in metastatic spinal tumour 
causing cord compression in a southern Chinese population. J Ort Trauma Rehab. 18(1):15-21

38. American Society of Anesthesiologists (1963) New classification of physical status. Anesthesiology 24:111
39. Luksanapruksa P, Buchowski JM, Hotchkiss W et al (2017) Prognostic factors in patients with spinal metastasis: a systematic review and meta-analysis. Spine J 17(5):689-708 